$4-25-2018$

\title{
Orderable groups, elementary theory, and the Kaplansky conjecture
}

\author{
Benjamin Fine \\ Fairfield University, fine@fairfield.edu \\ Anthony Gaglione \\ Gerhard Rosenberger \\ Dennis Spellman
}

Follow this and additional works at: https://digitalcommons.fairfield.edu/mathandcomputersciencefacultypubs

(C) 2018 Walter de Gruyter GmbH, Berlin/Boston

The final publisher PDF has been archived here with permission from the copyright holder.

\section{Peer Reviewed}

\section{Repository Citation}

Fine, Benjamin; Gaglione, Anthony; Rosenberger, Gerhard; and Spellman, Dennis, "Orderable groups, elementary theory, and the Kaplansky conjecture" (2018). Mathematics Faculty Publications. 58. https://digitalcommons.fairfield.edu/mathandcomputerscience-facultypubs/58

\section{Published Citation}

Fine, B., Gaglione, A., Rosenberger, G., \& Spellman, D. (2018). Orderable groups, elementary theory, and the Kaplansky conjecture. Groups Complexity Cryptology, 10(1), 43-52. https://doi.org/10.1515/gcc-2018-0005.

This Article is brought to you for free and open access by the Mathematics Department at DigitalCommons@Fairfield. It has been accepted for inclusion in Mathematics Faculty Publications by an authorized administrator of DigitalCommons@Fairfield. For more information, please contact digitalcommons@fairfield.edu. 


\title{
Research Article
}

\section{Benjamin Fine*, Anthony Gaglione, Gerhard Rosenberger and Dennis Spellman \\ Orderable groups, elementary theory, and the Kaplansky conjecture}

https://doi.org/10.1515/gcc-2018-0005

Received March 8, 2018

\begin{abstract}
We show that each of the classes of left-orderable groups and orderable groups is a quasivariety with undecidable theory. In the case of orderable groups, we find an explicit set of universal axioms. We then consider the relationship with the Kaplansky group rings conjecture and show that $\mathcal{K}$, the class of groups which satisfy the conjecture, is the model class of a set of universal sentences in the language of group theory. We also give a characterization of when two groups in $\mathcal{K}$ or more generally two torsion-free groups are universally equivalent.
\end{abstract}

Keywords: Orderable group, universal class, Kaplansky conjecture

MSC 2010: Primary 20E99; secondary 16S34, 20515

Dedicated to the memory of Seymour Lipschutz

\section{Introduction}

The first author is fond of using the positive solution of the Tarski conjecture on the elementary equivalence of the nonabelian free groups to give alternative proofs of results deduced for groups elementarily equivalent to $F=\left\langle a_{1}, a_{2} ;\right\rangle$, especially (with a few exceptions of low genus) surface groups (both orientable and nonorientable); with tongue in cheek, he calls such alternative proofs "something for nothing." (Of course, the proof of the Tarski conjecture is exceedingly nontrivial!) This is particularly striking in the case when the property to be deduced is, on the surface, anything but first-order, only to subsequently be found to be such after all. One such example is a theorem of Magnus asserting that if in a free group two elements $a$ and $b$ have the same normal closure, then $b$ must be either conjugate to $a$ or conjugate to $a^{-1}$. An explicit set of first-order sentences capturing the above theorem of Magnus may be found, for example, in [10] (see also [7, 8]). So we get "for free" that Magnus' theorem holds in surface groups (of sufficiently high genus). The authors of this paper attended the 2015 Zassenhaus Group Theory conference. At that conference, Zoran Sunic gave a talk on left relative convex subgroups. In this talk, he mentioned that surface groups (of sufficiently high genus) are orderable as well as the result (due independently to Iwasasa [12] and Neumann [17]) modulo the theorem of Magnus [15], asserting that a free group $F$ is residually nilpotent with torsion-free lower central quotients $F / \gamma_{n+1}(F)$ that free groups are orderable. That prompted the second author to pose the question, with the "something for nothing" approach in mind, of whether group orderability is a first-order property. Surpris-

\footnotetext{
*Corresponding author: Benjamin Fine, Department of Mathematics, Fairfield University, Fairfield, CT 06430, USA, e-mail: Ben1902@aol.com Anthony Gaglione, Department of Mathematics, United States Naval Academy, Annapolis, MD 21402, USA, e-mail: agaglione@aol.com. http://orcid.org/0000-0002-6435-6183

Gerhard Rosenberger, Fachbereich Mathematik, University of Hamburg, Bundesstr. 55, 20146 Hamburg, Germany, e-mail: gerhard.rosenberger@uni-math.hamburg.de

Dennis Spellman, Department of Statistics, Temple University, Philadelphia, PA 19122, USA, e-mail: spellman@temple.edu
} 
ingly the answer is positive. Thus, every elementarily free group is orderable. More is true. Since the axioms are universal sentences, every nonabelian universally free group is orderable. We further show that each of the classes of left-orderable groups and orderable groups is a quasivariety with undecidable theory. In the finitely generated case, universally free groups are precisely, in the terminology of Sela [20], the nonabelian limit groups. These groups are also called nonabelian fully residually free groups.

One reason that orderable groups are of interest is their ties to the Kaplansky group rings conjecture that a group ring $K[G]$ over a field $K$ has no zero divisors over a field $K$ if and only if $G$ is torsion-free. If $G$ is a group and the group ring $K[G]$ has no zero divisors for any field $K$, we call $G$ a Kaplansky group. We let $\mathcal{K}$ denote the class of Kaplansky groups. It is known that any orderable group is a Kaplansky group. Our results show that all elementary free groups, and in particular all universally free groups, are then Kaplansky groups. It was pointed out to us by the referee that our proof for orderable groups follows also for the groups in $\mathcal{K}$. We then prove in Section 4 that the class $\mathcal{K}$ is the model class of a set of universal sentences in the language $L_{0}$ appropriate for group theory. We thank the referee for pointing this out to us. We also prove a theorem which characterizes when two Kaplansky groups are universally equivalent.

In this paper, we unabashedly embrace the Axiom of Choice. In particular, Zermelo's well-ordering principle is available to us. Our ordinals will be von Neumann ordinals so that every ordinal coincides with the set of its predecessors. We let $\omega$ be the first limit ordinal which we identify with the set of nonnegative integers endowed with its natural order.

Definition 1.1. A group $G$ is left-orderable provided it admits a total order $\leq$ satisfying $h g_{1} \leq h g_{2}$ whenever $g_{1} \leq g_{2}$. Moreover, $G$ is orderable provided it admits a total order $\leq$ satisfying both

$$
\begin{array}{ll}
h g_{1} \leq h g_{2} & \text { whenever } g_{1} \leq g_{2}, \\
g_{1} h \leq g_{2} h & \text { whenever } g_{1} \leq g_{2} .
\end{array}
$$

Clearly, both left-orderability and orderability are inherited by subgroups.

Fixing notation, if $G$ is a group and $H$ and $K$ are (not necessarily distinct) subgroups in $G$, then $[H, K]$ shall be the subgroup of $G$ generated by all commutators $[h, k]=h^{-1} k^{-1} h k$ as $h$ and $k$ vary independently over $H$ and $K$, respectively. The lower central series of $G$ is defined recursively by $\gamma_{1}(G)=G$, and if $n>1$ and $\gamma_{n-1}(G)$ has already been defined, then $\gamma_{n}(G)=\left[\gamma_{n-1}(G), G\right]$.

Theorem $1.2([12,17])$. Suppose $G$ is a group such that

$$
\bigcap_{0 \leq n<\omega} \gamma_{n+1}(G)=\{1\}
$$

and $G / \gamma_{n+1}(G)$ is torsion-free for each $n, 0 \leq n<\omega$. Then $G$ is orderable.

Since, by a classical result of Magnus [15], the hypotheses of Theorem 1.2 are satisfied by free groups, we have the immediate corollary.

Corollary 1.3. Every free group is orderable.

\section{Preliminaries from logic}

Let $L_{0}$ be the first-order language with equality containing a binary operation symbol • (suppressed notationally in favor of juxtaposition), a unary operation symbol ${ }^{-1}$ and a constant symbol 1 . Thus, an $L_{0}$-structure is a set $G$ provided with a binary operation $G^{2} \rightarrow G,(g, h) \mapsto g h$, a unary operation $G \rightarrow G, g \mapsto g^{-1}$ and a distinguished element $1 \in G$.

A group is then an $L_{0}$-structure which is a model of the axioms

$$
\begin{aligned}
& \forall x_{1}, x_{2}, x_{3}\left(\left(x_{1} x_{2}\right) x_{3}=x_{1}\left(x_{2} x_{3}\right)\right), \\
& \forall x(x 1=x), \\
& \forall x\left(x x^{-1}=1\right) .
\end{aligned}
$$


Suppose $X=\left\{x_{n}: n<\omega\right\}$ is the set of variables of $L_{0}$. The set of terms of $L_{0}$ is defined recursively as follows: Every variable $x_{n}$ is a term; moreover, the constant symbol 1 is a term. If $t$ is a term already defined, then $(t)^{-1}$ is a term. If $\left(t_{1}, t_{2}\right)$ is an ordered pair of terms already defined, then $\left(t_{1}\right)\left(t_{2}\right)$ is a term. Modulo the group axioms, every term is equal to a word on the variables and their formal inverses. Here, 1 is identified with the empty word.

An identity or law of $L_{0}$ is a universal sentence of the form $\forall \bar{x}(T(\bar{x})=t(\bar{x}))$, where $\bar{x}$ is a tuple of distinct variables and $T(\bar{x})$ and $t(\bar{x})$ are terms of $L_{0}$ involving at most the variables in $\bar{x}$. Thus, for example, the group axioms are identities of $L_{0}$.

A quasi-identity of $L_{0}$ is a universal sentence of the form

$$
\forall \bar{x}\left(\bigwedge_{i}\left(S_{i}(\bar{x})=s_{i}(\bar{x})\right) \rightarrow(T(\bar{x})=t(\bar{x}))\right),
$$

where $\bar{x}$ is a tuple of distinct variables and $S_{i}(\bar{x}), s_{i}(\bar{x}), T(\bar{x})$, and $t(\bar{x})$ are terms of $L_{0}$ involving at most the variables in $\bar{x}$. Note that the identity $\forall \bar{x}(T(\bar{x})=t(\bar{x}))$ is equivalent to the quasi-identity $\forall \bar{x}((1=1) \rightarrow(T(\bar{x})=t(\bar{x})))$, so that identities may be considered as special cases of quasi-identities. Note also that, modulo the group axioms, the quasi-identity

$$
\forall \bar{x}\left(\bigwedge_{i}\left(S_{i}(\bar{x})=s_{i}(\bar{x})\right) \rightarrow(T(\bar{x})=t(\bar{x}))\right)
$$

is equivalent to one of the form

$$
\forall \bar{x}\left(\bigwedge_{i}\left(u_{i}(\bar{x})=1\right) \rightarrow(w(\bar{x})=1)\right),
$$

where the $u_{i}(\bar{x})$ and $w(\bar{x})$ are words in at most the variables in $\bar{x}$ and their formal inverses. A quasivariety of groups is the model class of a set of quasi-identities of $L_{0}$ together with the group axioms. Following Chang and Keisler [6], we call a class of $L_{0}$-structures elementary provided it is the model class of at least one set of sentences of $L_{0}$. A theorem of Mal'cev [16] asserts that a nonempty elementary class of groups is a quasivariety of groups if and only if it is closed under taking subgroups and (unrestricted) direct products.

Two (not necessarily distinct) $L_{0}$-structures $G$ and $H$ are elementarily equivalent, in symbols $G \equiv H$, provided they satisfy precisely the same sentences of $L_{0}$. (In particular, if $G \equiv H$, then $H$ is a group if and only if $G$ is a group.) The next theorem gives an algebraic characterization of elementary equivalence. It was initially proven by Keisler assuming the Generalized Continuum Hypothesis and subsequently proven by Shelah without need of that assumption.

Theorem 2.1 ([21]). Two $L_{0}$-structures are elementarily equivalent if and only if they have isomorphic ultrapowers.

For a discussion of ultraproducts, see, for example, [6].

Theorem 2.2 ([6]). A class of $L_{0}$-structures is an elementary class if and only if it is closed under both elementary equivalence and ultraproducts.

We shall have occasion to apply the following classical "trick".

Theorem 2.3. Let $X$ be an elementary class of groups. If $X$ contains a finitely presented group with unsolvable word problem, then the theory of $X$ is undecidable.

Proof. Suppose $G$, lying in $X$, has finite presentation

$$
\left\langle a_{1}, \ldots, a_{m} ; R_{1}\left(a_{1}, \ldots, a_{m}\right)=\cdots=R_{n}\left(a_{1}, \ldots, a_{m}\right)=1\right\rangle
$$

and has unsolvable word problem. For each word $w\left(x_{1}, \ldots, x_{m}\right)$ on the distinct variables $x_{1}, \ldots, x_{m}$ and their formal inverses, let $\sigma_{w}$ be the sentence

$$
\forall x_{1}, \ldots, x_{m}\left(\bigwedge_{i=1}^{n}\left(R_{i}\left(x_{1}, \ldots, x_{m}\right)=1\right) \rightarrow\left(w\left(x_{1}, \ldots, x_{m}\right)=1\right)\right) .
$$


If there were a recursive algorithm to decide whether or not each $\sigma_{w}$ is true in every group in $X$, then we would have an algorithm to solve the word problem for $G$. The contradiction shows that the theory of $X$ is undecidable.

Finally, in this section we explicitly mention the positive solution to the Tarski question.

Theorem $2.4([13,20])$. Every nonabelian free group is elementarily equivalent to $F=\left\langle a_{1}, a_{2} ;\right\rangle$.

\section{Orderable groups as a universal quasivariety}

We follow the notation in Passman's book [18]. Let $G$ be a group and let $S$ be a subsemigroup of $G$. Just as for subgroups, we call $S$ normal in $G$ provided it is invariant under conjugation by arbitrary elements in $G$. If $n$ is a positive integer and $\left(b_{1}, \ldots, b_{n}\right) \in G^{n}$, we let $S G\left(b_{1}, \ldots, b_{n}\right)$ be the least normal subsemigroup of $G$ containing $\left\{b_{1}, \ldots, b_{n}\right\}$ as a subset, and let $S\left(b_{1}, \ldots, b_{n}\right)$ be the least subsemigroup of $G$ containing $\left\{b_{1}, \ldots, b_{n}\right\}$ as a subset.

Continuing to fix notation, we let $\mathbb{Z}$ be the ring of integers, let $\mathbb{N}=\{1,2,3, \ldots\}$ be its positive class and let $U=\{1,-1\}$ be its group of units.

Theorem 3.1 (Passman [18]). The following statements hold:

(i) A necessary and sufficient condition for a group $G$ to be left orderable is that, for every finite subset $\left\{a_{1}, \ldots, a_{n}\right\}$ of $G \backslash\{1\}$, the intersection of the $2^{n}$ semigroups $S\left(a_{1}^{\varepsilon_{1}}, \ldots, a_{n}^{\varepsilon_{n}}\right)$ is empty as $\left(\varepsilon_{1}, \ldots, \varepsilon_{n}\right)$ varies over $U^{n}$.

(ii) A necessary and sufficient condition for a group $G$ to be orderable is that, for every finite subset $\left\{a_{1}, \ldots, a_{n}\right\}$ of $G \backslash\{1\}$, the intersection of the $2^{n}$ normal subsemigroups $S G\left(a_{1}^{\varepsilon_{1}}, \ldots, a_{n}^{\varepsilon_{n}}\right)$ is empty as $\left(\varepsilon_{1}, \ldots, \varepsilon_{n}\right)$ varies over $U^{n}$.

Theorem 3.2. The class of orderable groups is elementary.

Proof. For each $n \in \mathbb{N}, \bar{\varepsilon}=\left(\varepsilon_{1}, \ldots, \varepsilon_{n}\right) \in U^{n}$ and each $\bar{N}=\left(N_{0}, N_{1}, \ldots, N_{n}\right) \in \mathbb{N}^{n+1}$, let $w(\bar{\varepsilon}, \bar{N})$ be a word of positive length at most $N_{0}$ on the free semigroup generators (regarded as compound symbols, so no formal cancellation is permitted) $z_{i, j}^{-1} y_{i}^{\varepsilon_{i}} z_{i, j}, 1 \leq i \leq n, 1 \leq j \leq N_{i}$. In view of Lorenzen's theorem (Theorem 3.1 above), the class of orderable groups is axiomatized by the group axioms together with the sentences

$$
\forall x, y_{1}, \ldots, y_{n}, z_{1,1}, \ldots, z_{n, N_{n}}\left(\left(\bigwedge_{1 \leq i<j \leq n}\left(y_{i} \neq y_{j}\right) \wedge \bigwedge_{\bar{\varepsilon} \in U^{n}}(x=w(\bar{\varepsilon}, \bar{N}))\right) \rightarrow \bigvee_{i=1}^{n}\left(y_{i}=1\right)\right)
$$

as $n$ varies over $\mathbb{N}$, and the $\bar{N}$ vary over $\mathbb{N}^{n+1}$, and as the $w(\bar{\varepsilon}, \bar{N})$ vary over all possible choices (note that $a_{i} \neq 1$, so $y_{i} \neq 1$ ).

Corollary 3.3. Any elementary free group, and more generally any universally free group, is orderable.

Theorem 3.4. The class of left-orderable groups is elementary.

Proof. As in the proof of Theorem 3.2, we give an explicit set of sentences which describes this class of groups. These follow from the two parts of Theorem 3.1. To capture the class of left-orderable groups, we need the group axioms together with the sentences, one for each $n \in \mathbb{N}$ with

$$
\bar{\varepsilon}=\left(\varepsilon_{1}, \ldots, \varepsilon_{n}\right) \in U^{n},
$$

and each $N_{0} \in \mathbb{N}$,

$$
\forall x, y_{1}, \ldots, y_{n}\left(\bigwedge_{1 \leq i<j \leq n}\left(y_{i} \neq y_{j}\right) \wedge \bigwedge_{\bar{\varepsilon}} \in U^{n}\left(x=u\left(\bar{\varepsilon}, N_{0}\right)\right) \rightarrow \bigvee_{i=1}^{n}\left(y_{i}=1\right)\right),
$$

where $u\left(\bar{\varepsilon}, N_{0}\right)$ is a semigroup word. 
Theorem 3.5. The class of orderable groups is a quasivariety of groups with an undecidable theory.

Proof. In view of Mal'cev's characterization of quasivarieties, it suffices to show that the class of orderable groups is closed under taking subgroups and unrestricted direct products. We have already noted that order is inherited by subgroups. (Alternatively, since the class has a set of universal axioms, it is closed under taking substructures.)

Suppose $\leq_{0}$ is an order on the group $G_{0}$ and $\leq_{1}$ is an order on the group $G_{1}$. Then the lexicographic order on $G_{0} \times G_{1}$ (i.e., if $\left(g_{0}, g_{1}\right) \neq\left(h_{0}, h_{1}\right)$, then $\left(g_{0}, g_{1}\right)<\left(h_{0}, h_{1}\right)$ provided either $g_{0}<{ }_{0} h_{0}$ or $g_{0}=h_{0}$ and $\left.g_{1}<{ }_{1} h_{1}\right)$ makes $G_{0} \times G_{1}$ into an ordered group. Now, [11, Section 47, p. 292, Corollary 2 of Chapter 7] asserts that if an elementary class $X$ is closed under the direct product of two structures, then it is closed under arbitrary direct products of nonvoid families of structures in $x$.

Alternatively, we could argue as follows: We may well-order the index set of any nonvoid family of orderable groups. There is no loss of generality in taking the index set to be an ordinal $\alpha$. Suppose $\leq \xi$ is a left order on $G_{\xi}$ for all ordinals $\xi<\alpha$. Then the lexicographical order on

$$
G=\prod_{\xi<\alpha} G_{\xi}
$$

(i.e., if $\left(g_{\xi}\right)_{\xi<\alpha} \neq\left(h_{\xi}\right)_{\xi<\alpha}$, then $\left(g_{\xi}\right)_{\xi<\alpha}<\left(h_{\xi}\right)_{\xi<\alpha}$ provided $g_{\mu}<_{\mu} h_{\mu}$, where $\mu$ is the least ordinal $\xi$ such that $g_{\xi} \neq h_{\xi}$ ) makes $G$ into an ordered group. It follows that the class of orderable groups is a quasivariety of groups.

Theorem 3.6. The class of left-orderable groups is a quasivariety of groups.

Theorem 3.7. The theory of orderable groups is undecidable.

Proof. By a result of Bludov and Glass [4], there is a finitely presented orderable group with unsolvable word problem. The result then follows from the proof of Theorem 2.3.

Exactly the same proof shows the following theorem.

Theorem 3.8. The theory of left-orderable groups is undecidable.

Remark 3.9. It would be of interest to find explicit quasi-identities axiomatizing each of the classes of orderable groups and left-orderable groups.

\section{The class $\mathcal{K}$ of groups satisfying the Kaplansky conjecture}

The Kaplansky conjecture for group rings is that the group ring $K[G]$ over a field $K$ has no zero divisors if and only if $G$ is torsion-free. A stronger conjecture that implies this one is the Kaplansky unit conjecture that in a group ring $K[G]$, with $K$ being a field and $G$ a torsion-free group, the only units are the trivial ones of the form $u$, where $u \in K \backslash\{0\}$ and $g \in G$.

Both conjectures are still open in general but the basic group rings conjecture has been shown to hold in many classes of groups. In particular, $K[G]$ has no zero divisors if $G$ is orderable or left-orderable. We let $\mathcal{K}$ denote the class of groups for which the Kaplansky conjecture is true, that is, the class of groups $G$ such that $K[G]$ has no zero divisors for any field $K$. If $G \in \mathcal{K}$, we call $G$ a Kaplansky group. From our results on orderable groups and the fact that $G \in \mathcal{K}$ for any orderable group $K$, it follows that an elementary free group and more generally any universally free group is in $\mathcal{K}$. It was pointed out to us by the referee that our techniques in handling orderable groups can also be applied directly to the class $\mathcal{K}$. We show that the class $\mathcal{K}$ is the model class of a set of universal sentences of $L_{0}$. We thank the referee for pointing this out to us.

We first note that the fact that $G \in \mathcal{K}$ if $G$ is finitely generated and elementary can be proved directly using the Gaglione-Spellman-Remeslennikov characterization of finitely generated universally free groups as precisely the finitely generated nonabelian fully residually free groups (see [7]). 
Lemma 4.1. Let $G$ be a finitely generated fully residually free group. Then $G \in \mathcal{K}$, that is, $K[G]$ has no zero divisors for any field $K$.

Proof. First, let us recall that a group $G$ is fully residually free if for any finite set of nontrivial elements $\left\{g_{1}, \ldots, g_{n}\right\} \subset G \backslash\{1\}$ there exists a epimorphism $\phi: G \rightarrow F$ where $F$ is a free group and $\phi\left(g_{i}\right) \neq 1$ for all $i=1, \ldots, n$. A result of Gaglione and Spellman, and independently Remeslennikov, (see [7]) characterizes the finitely generated universally free groups as precisely the finitely generated fully residually free groups. Now let $G$ be fully residually free and $K$ a field. Suppose that $z_{1}=f_{1} g_{1}+\cdots+f_{n} g_{n} \in K[G]$ and $z_{2}=k_{1} h_{1}+\cdots+k_{m} h_{m} \in K[G]$, and assume that $z_{1} \neq 0$ and $z_{2} \neq 0$. We may assume that $f_{i} \neq 0$ for $i=1, \ldots, n, g_{i} g_{j}^{-1} \neq 1$ for $1 \leq i<j \leq n$, and that $k_{i} \neq 0$ for $i=1, \ldots, m_{j}, h_{i} h_{j}^{-1} \neq 1$ for $1 \leq i<j \leq m$. Suppose that $z_{1} z_{2}=0$ in $K[G]$. Then there exists a homomorphism $\phi: G \rightarrow F$ with $F$ being a free group and $\phi\left(g_{i} g_{j}^{-1}\right) \neq 1$ for $1 \leq i<j \leq m$ and $\phi\left(h_{i} h_{j}^{-1}\right) \neq 1$ for $1 \leq i<j \leq m$. The homomorphism $\phi$ extends to a group ring homomorphism $\phi^{*}: K[G] \rightarrow K[F]$ with $\phi^{*}\left(z_{1}\right) \neq 0$ and $\phi^{*}\left(z_{2}\right) \neq 0$. We then have $\phi^{*}\left(z_{1} z_{2}\right)=0$, which is a contradiction since $K[F]$ has no zero divisors with $F$ being a free group. Therefore, $K[G]$ has no zero divisors.

The above lemma also follows from the following argument: It is known that groups universally equivalent to free groups are orderable since they are subgroups of ultrapowers of a free nonabelian group, which is orderable. Hence any group universally equivalent to a free group is a Kaplansky group.

We now prove that $\mathcal{K}$ is a universal class.

Theorem 4.2. Let $\mathcal{K}$ be the class of groups for which the Kaplansky group ring conjecture is true. Then $\mathcal{K}$ is the model class of a set of universal sentences of $L_{0}$.

Proof. From [1, Chapter 7, Theorem 3.10] a class of group $X$ is the model class of a set of sentences in $L_{0}$ if and only if $X$ is closed under isomorphisms and ultraproducts, and its complement $c X$ within the set of $L_{0}$-structures is closed under ultrapowers.

Now let $\mathcal{K}$ be the class of groups for which the Kaplansky group ring conjecture is true. Clearly, $G_{1} \cong G_{2}$ implies that $K\left[G_{1}\right] \cong K\left[G_{2}\right]$, so $\mathcal{K}$ is closed under isomorphisms. Now let $\Lambda$ be a nonempty index set and let $\left(G_{\lambda}\right)_{\lambda \in \Lambda}$ be a family of groups of $\mathcal{K}$. Let $D$ be an ultrafilter on $\Lambda$. If $A \subseteq \Lambda$, let $A^{\prime}=\Lambda / A$ be its complement in $\Lambda$. Let $\mathcal{U}$ be the ultraproduct constructed from the above data. We show that $K[\mathcal{U}]$ has no zero divisors for every field $K$.

Suppose that $a, b \in K[\mathcal{U}]$ and $a b=0$. We show that either $a=0$ or $b=0$. Let

$$
a=\sum_{i=1}^{m} \alpha_{i} \zeta_{i} \quad \text { and } \quad b=\sum_{j=1}^{n} \beta_{j} \eta_{j}
$$

where

$$
\begin{aligned}
\zeta_{i} & =\left[\left(x_{i, \lambda}\right)_{\lambda \in \Lambda}\right]_{D}, \quad i=1, \ldots, m, \\
\eta_{j} & =\left[\left(y_{j, \lambda}\right)_{\lambda \in \Lambda}\right]_{D}, \quad j=1, \ldots, n .
\end{aligned}
$$

Now let

$$
A=\left\{\lambda \in \Lambda:\left(\sum_{i=1}^{n} \alpha_{i} x_{i, \lambda}\right)\left(\sum_{j=1}^{m} \beta_{j} \eta_{j, \lambda}\right)=0\right\} .
$$

Then $A \in D$. For each $\lambda \in A$ we must have either

$$
\sum_{i=1}^{m} \alpha_{i} x_{i, \lambda}=0 \quad \text { or } \quad \sum_{j=1}^{m} \beta_{j} y_{j, \lambda}=0 .
$$

Therefore, if

$$
B=\left\{\lambda \in \Lambda: \sum_{i=1}^{m} \alpha_{i} x_{i, \lambda}=0\right\} \quad \text { and } \quad C=\left\{\lambda \in \Lambda: \sum_{j=1}^{n} \beta_{j} y_{j, \lambda}=0\right\}
$$


then

$$
B \cup C \in D \text { since } B \cup C=A .
$$

Suppose that $B \notin D$ and $C \notin D$. Then

$$
B^{\prime} \in D \text { and } C^{\prime} \in D,
$$

and therefore $B^{\prime} \cap C^{\prime} \in D$. Then $B^{\prime} \cap C^{\prime}=(B \cup C)^{\prime} \notin D$ since $B \cup C \in D$. This contradiction shows that $B \in D$ or $C \in D$. If $B \in D$, then $a=0$, while if $C \in D$, then $b=0$. From this it follows that the group ring $K[\mathcal{U}]$ for the ultraproduct $U$ has no zero divisors.

Now let $G$ be a group not in $\mathcal{K}$ and let $* G$ be an ultrapower of $G$. If $* G$ is not a group, then $G$ violates one of the group axioms, and then $* G$ also violates this axiom and $* G \notin \mathcal{K}$. If $G$ is a group but not a Kaplansky group, then $K[G]$ has zero divisors for some field $K$. The embedding $G \rightarrow * G$ induces an embedding $K[G] \rightarrow K[* G]$ so that $K[* G]$ has zero divisors and $* G \notin \mathcal{K}$. It follows now from the result in Bell and Slomson [1] that $\mathcal{K}$ is the model class of a set of sentences of $L_{0}$.

Suppose that $G \in \mathcal{K}$ and $G_{0} \subset G$. Since for any field $K$ the group ring $K[G]$ contains no zero divisors, the group ring $K\left[G_{0}\right]$ cannot contain zero divisors. Hence $\mathcal{K}$ is closed under subgroups.

Now, [6, Theorem 5.2.4] asserts that a theory $\mathcal{T}$ is closed under submodels if and only if $\mathcal{T}$ has a set of universal axioms. Therefore, $\mathcal{K}$ is the model class of a set of universal sentences of $L_{0}$, thus proving the theorem.

We now prove a technical theorem that characterizes when two torsion-free groups, and in particular two Kaplansky groups, are universally equivalent in terms of Horn sentences in their integral group rings (see $[7,10])$. In what follows, $L_{0}$ is a first-order language appropriate for group theory, $L_{1}$ is a first-order language appropriate for ring theory, and $L_{2}$ a first-order language appropriate for group-rings (see [9]). For the proof of this theorem we need the following lemma.

Lemma 4.3. Let $G$ be a torsion-free group and $g$ a nontrivial element of $G$. Then $1-g$ is not a zero divisor in the group ring $\mathbb{Z}[G]$

Proof. We prove the stronger result that if $g$ is an element of infinite order in any group $G$, then $1-g$ is not a zero divisor in $\mathbb{Z}[G]$.

Suppose that $g$ has infinite order. Consider the additive endomorphism $\phi: \mathbb{Z}[G] \rightarrow \mathbb{Z}[G]$ given by $\phi(x)=g x$.

We claim that $\phi$ has no fixed point $x_{0} \neq 0$. Suppose that $\phi\left(x_{0}\right)=x_{0}$. Write $x_{0}=m_{1} g_{1}+\cdots+m_{k} g_{k}$, where $m_{i} \in \mathbb{Z} \backslash\{0\}$ for $i=1, \ldots, k$ and the $g_{1}, \ldots, g_{k}$ are distinct elements of $G$.

Then $x_{0}=\phi\left(x_{0}\right)=m_{1} g g_{1}+\cdots+m_{k} g g_{k}$. Since the $g g_{c}$ are distinct, it follows that $g g_{1}, \ldots, g g_{k}$ must be $g_{1}, \ldots, g_{k}$ in some order by uniqueness of the representation. Thus the restriction of $\phi$ to $\left\{g_{1}, \ldots, g_{k}\right\}$ is a permutation $\pi$.

Suppose that $\pi$ has order $n$. Then in particular $g^{n} g_{1}=g_{1}$, so $g^{n}=1$, contradicting the assumption that $g$ has infinite order. Therefore, $\phi$ has no nonzero fixed points.

A similar argument shows that $\psi: \mathbb{Z}[G] \rightarrow \mathbb{Z}[G]$ given by $\psi(x)=x g$ has no nonzero fixed points.

Now suppose that $1-g$ were a zero divisor in $\mathbb{Z}[G]$. Then there would be $x_{0} \in \mathbb{Z}[G] \backslash\{0\}$ such that either $(1-g) x_{0}=0$ or $x_{0}(1-g)=0$. In the first case $x_{0}$ would be a fixed point of $\phi$, and in the second case $x_{0}$ would be a fixed point of $\psi$. This contradiction shows that $1-g$ is not a zero divisor in $\mathbb{Z}[G]$.

Now we give the technical theorem.

Theorem 4.4. Let $G$ and $H$ be torsion-free groups. Then $H$ is a model of those universal sentences of $L_{0}$ true in $G$ if and only if the group ring $\mathbb{Z}[H]$ satisfies every strict universal Horn sentence of $L_{2}$ of the form

$$
\forall \bar{x}\left(\left(\bigwedge_{k=1}^{n} \Gamma\left(x_{k}\right) \wedge \bigwedge_{i=1}^{p}\left(u_{i}(\bar{x})=1\right)\right) \rightarrow\left(\left(1-w_{1}(\bar{x})\right) \cdots\left(1-w_{q}(\bar{x})\right)=0\right)\right)
$$

true in the group ring $\mathbb{Z}[G]$, where $\bar{x}=\left(x_{1}, \ldots, x_{n}\right)$ is a tuple of distinct variables and the $u_{i}(\bar{x})$ and $w_{j}(\bar{x})$ are group words on the variables and their formal inverses. 
Proof. Suppose that $\mathbb{Z}[H]$ satisfies every universal Horn sentence in $L_{2}$ of the form

$$
\forall \bar{x}\left(\left(\bigwedge_{k=1}^{n} \Gamma\left(x_{k}\right) \wedge \bigwedge_{i=1}^{p}\left(u_{i}(\bar{x})=1\right)\right) \rightarrow\left(\left(1-w_{1}(\bar{x})\right) \cdots\left(1-w_{q}(\bar{x})\right)=0\right)\right)
$$

true in $\mathbb{Z}[G]$.

To deduce a contradiction, assume that $H$ violates some universal sentence of $L_{0}$ true in $G$. Then $H$ satisfies some existential sentence of $L_{0}$ false in $G$. The usual argument then shows that $H$ satisfies some primitive sentence of $L_{0}$ false in $G$. Let this primitive sentence be

$$
\exists \bar{x}\left(\bigwedge_{i=1}^{p}\left(u_{i}(\bar{x})=1\right) \wedge \bigwedge_{j=1}^{q}\left(w_{j}(\bar{x}) \neq 1\right)\right)
$$

Since (4.1) is false in $G$, the group $G$ satisfies

$$
\forall \bar{x}\left(\bigwedge_{i=1}^{p}\left(u_{i}(\bar{x})=1\right) \rightarrow \bigvee_{j=1}^{q}\left(w_{j}(\bar{x})=1\right)\right)
$$

Since $\mathbb{Z}[G]$ has no zero divisors of the form $1-g$ by Lemma $4.3, \mathbb{Z}[G]$ satisfies

$$
\forall \bar{x}\left(\left(\bigwedge_{k=1}^{n} \Gamma\left(x_{k}\right) \wedge \bigwedge_{i=1}^{p}\left(u_{i}(\bar{x})=1\right)\right) \rightarrow\left(\left(1-w_{1}(\bar{x})\right) \cdots\left(1-w_{q}(\bar{x})\right)=0\right)\right)
$$

By hypothesis, $\mathbb{Z}[H]$ satisfies (4.2). Since $\mathbb{Z}[H]$ has no zero divisors of the form $1-g$, the group $H$ satisfies

$$
\forall \bar{x}\left(\bigwedge_{i=1}^{p}\left(u_{i}(\bar{x})=1\right) \rightarrow \bigvee_{j=1}^{q}\left(w_{j}(\bar{x})=1\right)\right)
$$

But that contradicts the assumption that $H$ satisfies

$$
\exists \bar{x}\left(\bigwedge_{i=1}^{p}\left(u_{i}(\bar{x})=1\right) \wedge \bigwedge_{j=1}^{q}\left(w_{j}(\bar{x}) \neq 1\right)\right) .
$$

The contradiction shows that $H$ is a model of those universal sentences of $L_{0}$ true in $G$.

Conversely, suppose that $H$ is a model of those universal sentences of $L_{0}$ true in $G$. Now suppose that

$$
\forall \bar{x}\left(\left(\bigwedge_{i=1}^{k}\left(\Gamma\left(x_{i}\right) \wedge \bigwedge_{i=1}^{p}\left(u_{i}(\bar{x})\right)=1\right)\right) \rightarrow\left(\left(1-w_{1}(\bar{x})\right) \cdots\left(1-w_{q}(\bar{x})\right)=0\right)\right)
$$

is true in $\mathbb{Z}[G]$. Then, since $\mathbb{Z}[G]$ has no zero divisors of the form $1-g$,

$$
\forall \bar{x}\left(\left(\bigwedge_{i=1}^{p}\left(u_{i}(\bar{x}=1)\right) \rightarrow \bigvee_{j=1}^{q}\left(w_{j}(\bar{x})\right)=1\right)\right)
$$

is true in $G$.

Since $H$ satisfies those universal sentences of $L_{0}$ true in $G$, it follows that $H$ satisfies

$$
\forall \bar{x}\left(\bigwedge_{i=1}^{p}\left(u_{i}(\bar{x})=1\right) \rightarrow \bigvee_{j=1}^{q}\left(w_{j}(\bar{x})=1\right)\right) .
$$

Since $\mathbb{Z}[H]$ has no zero divisors of the form $1-g$, the group ring $\mathbb{Z}[H]$ satisfies

$$
\forall \bar{x}\left(\left(\bigwedge_{k=1}^{n} \Gamma\left(x_{k}\right) \wedge \bigwedge_{i=1}^{p}\left(u_{i}(\bar{x})=1\right)\right) \rightarrow\left(\left(1-w_{1}(\bar{x})\right) \cdots\left(1-w_{q}(\bar{x})\right)=0\right)\right) .
$$

Thus $G$ and $H$ are universally equivalent if and only if $\mathbb{Z}[G]$ and $\mathbb{Z}[H]$ satisfy the same given types of Horn sentences. 
From this we immediately get the following corollary.

Corollary 4.5. Let $G$ and $H$ be torsion-free groups. Then $G$ and $H$ are universally equivalent with respect to $L_{0}$ if and only if the group rings $\mathbb{Z}[G]$ and $\mathbb{Z}[H]$ satisfy the same strict universal Horn sentences of $L_{2}$ of the form

$$
\forall \bar{x}\left(\left(\bigwedge_{k=1}^{n} \Gamma\left(x_{k}\right) \wedge \bigwedge_{i=1}^{p}\left(u_{i}(\bar{x})=1\right)\right) \rightarrow\left(\left(1-w_{1}(\bar{x})\right) \cdots\left(1-w_{q}(\bar{x})\right)=0\right)\right)
$$

where $\bar{x}=\left(x_{1}, \ldots, x_{n}\right)$ is a tuple of distinct variables and the $u_{i}(\bar{x})$ and $w_{j}(\bar{x})$ are group words on the variables and their formal inverses.

We close this section and the paper by recalling some additional facts about the class $\mathcal{K}$ and then posing two questions.

It is known that any unique product group is in $\mathcal{K}$ (see [3]). In [3], Bowditch introduced a geometric variation of the unique product property called diffuse groups. Bowditch then discusses diffuse groups and fundamental groups of hyperbolic manifolds. He also provides an example of a group that is diffuse but not right-orderable. Recall that a group $G$ is locally indicable if every finitely generated nontrivial subgroup of $G$ has an infinite cyclic quotient. Burns and Hale [5] showed that locally indicable groups are right-orderable. However, Bergman [2] showed that a right-orderable group need not be locally indicable, answering a question posed by Howie. Any finite extension of a polycyclic group is right-orderable if and only if it is locally indicable [19], while other examples where this is true were examined by Longobardi, Maj and Rhemtulla [14] Another example of a right-orderable group that is not locally indicable is provided by the braided Thompson group $\mathcal{T}$, which is known to be right-orderable (see [2]), but has a simple subgroup of finite index so cannot have any infinite cyclic quotient, and hence cannot be locally indicable.

We close with two questions motivated by our results on orderable groups.

(1) Can we determine an explicit set of universal axioms for $\mathcal{K}$ ?

(2) Does the universal class $\mathcal{K}$ form a quasivariety?

\section{References}

[1] J. L. Bell and A. B. Slomson, Models and Ultraproducts: An Introduction, North-Holland, Amsterdam, 1969.

[2] G. M. Bergman, Right orderable groups that are not locally indicable, Pacific J. Math. 147 (1991), no. 2, 243-248.

[3] B. Bowditch, A variation of the unique product property, J. Lond. Math. Soc. (2) 62, (2000), no. 3, 813-826.

[4] V. V. Bludov and A. M. W. Glass, A finitely presented orderable group with insoluble word problem, Bull. Lond. Math. Soc. 44 (2012), no. 1, 85-98.

[5] R. G. Burns and V. W. D. Hale, A note on group rings of certain torsion-free groups, Canad. Math. Bull. 15 (1972), 441-445.

[6] C. C. Chang and H. J. Keisler, Model Theory, 2nd ed., North-Holland, Amsterdam, 1977.

[7] B. Fine, A. Gaglione, G. Rosenberger and D. Spellman, On elementary free groups, in: Algorithmic Problems of Group Theory, Their Complexity, and Applications to Cryptography, Contemp. Math. 633, American Mathematical Society, Providence (2015), 41-58.

[8] B. Fine, A. Gaglione, G. Rosenberger and D. Spellman, Something for nothing: Some consequences of the solution of the Tarski problems, in: Groups St. Andrews 2013, London Math. Soc. Lecture Note Ser. 422, Cambridge Univ. Press, Cambridge (2015), 242-270.

[9] B. Fine, A. Gaglione, G. Rosenberger and D. Spellman, The Tarksi theorems and elementary equivalence of group rings, Adv. Pure Math. 7 (2017), no. 2, 199-212.

[10] A. M. Gaglione, S. Lipschutz and D. Spellman, Almost locally free groups and a theorem of Magnus: Some questions, Groups Complex. Cryptol. 1 (2009), no. 2, 181-198.

[11] G. Grätzer, Universal Algebra, Van Nostrand, Princeton, 1968.

[12] K. Iwasawa, On linearly ordered groups, J. Math. Soc. Japan 1 (1948), 1-9.

[13] O. Kharlampovich and A. Myasnikov, Elementary theory of free non-abelian groups, J. Algebra 302 (2006), no. 2, 451-552.

[14] P. Longobardi, M. Maj and A. Rhemtulla, When is a right orderable group locally indicable?, Proc. Amer. Math. Soc. 128 (2000), no. 3, 637-641.

[15] W. Magnus, A. Karrass and D. Solitar, Combinatorial Group Theory: Presentations of Groups in Terms of Generators and Relations, Interscience, New York, 1966. 
[16] A. I. Mal'cev, Some remarks on quasi-varieties of algebraic structures, Algebra Logic 5 (1966), no. 3, 3-9.

[17] B. H. Neumann, On ordered groups, Amer. J. Math. 71 (1949), 1-18.

[18] D. S. Passman, The Algebraic Structure of Group Rings, Dover, New York, 2011.

[19] A. H. Rhemtulla, Polycyclic right-ordered groups, in: Algebra (Carbondale 1980), Lecture Notes in Math. 848, Springer, Berlin (1981), 230-234.

[20] Z. Sela, Diophantine geometry over groups. VI. The elementary theory of a free group, Geom. Funct. Anal. 16 (2006), no. 3 , 707-730.

[21] S. Shelah, Every two elementarily equivalent models have isomorphic ultrapowers, Israel J. Math. 10 (1971), $224-233$. 